\title{
Occlusion of an Incidentally-Found Dissecting Aneurysm of the Vertebral Artery at Removal of a Jugular Tubercle Meningioma
}

\author{
Yasuhiko Hayashi' ${ }^{12 *}$, Makoto Kimura ${ }^{1}$, Akira Kinoshita ${ }^{1}$ and Jun-ichiro Hamada ${ }^{2}$ \\ ${ }^{1}$ Department of Neurosurgery, Komatsu Municipal Hospital, Komatsu, Japan \\ ${ }^{2}$ Department of Neurosurgery, Graduate School of Medical Science, Kanazawa University, Japan
}

\begin{abstract}
This report presents a rare case of a 52-year-old female who had a left jugular tubercle meningioma with intratumoral hemorrhage and an adjacent dissecting aneurysm of Vertebral Artery (VA). The findings of neuroradiological images, such as fusiform dilatation and retention of contrast medium on angiogram and intramural hematoma on magnetic resonance imaging made a diagnosis of dissecting aneurysm. Because we speculated that the tumor was symptomatic and the dissecting aneurysm was not, only the removal of tumor was performed via lateral suboccipital craniectomy to result in total removal. Besides, intraoperatively, the intramural hematoma of dissecting aneurysm looked old and unruptured, we left it untreated. However, postoperative angiography revealed the occlusion of VA including the dissecting aneurysm. It was assumed the manipulation during the tumor removal altered the hemodynamics and promoted the thrombogenesis of VA with dissecting aneurysm.
\end{abstract}

Keywords: Dissecting aneurysm; Vertebral artery; Meningioma; Occlusion

\section{Introduction}

Commonly, once the dissection of vertebral artery (VA) occurs, subarachnoid hemorrhage or ischemic change happens with severe headache or neurological deficits [1-3]. However, there are some cases of dissecting aneurysm with headache alone or no symptoms $[1,2,4]$. Although conservative therapy is recommended, natural history of such dissecting aneurysms remains unclear. Therefore, periodic follow-up of neuroimaging is necessary to detect the changes leading to extension of dissection earlier $[5,6]$.

Our case presented a very rare co-localization of a dissecting aneurysm of VA and an adjacent jugular tubercle meningioma. The latter one became symptomatic due to intratumoral hemorrhage. An occlusion of both dissecting aneurysm and VA occurred after tumor removal. It was suggested that the hemodynamic change by intraoperative manipulation for the tumor and surrounding edematous brain causes the occlusion of dissecting aneurysm [3-6].

\section{Case Report}

This 52-year-old hypertensive woman was referred to our hospital because of severe headache. Neurological examination revealed nystagmus and ataxia on the left side. Magnetic resonance imaging (MRI) showed a round mass attached to the jugular tubercle; it was iso-intense and marked contrast enhanced with gadolinium; on T1weighted images the mass included an area of hypo-intensity. These findings led us to suspect jugular tubercle meningioma with acute intra-tumoral hemorrhage as the etiology of her headache. The left VA exhibited hyper-intensity along a flow void sign indicative of intramural hemorrhage in the vicinity of the tumor (Figure 1). The diameter of the right and left VA was symmetric.

A left vertebral angiogram revealed fusiform dilation in the arterial phase (Figure 2A \& Figure 2B) and contrast retention in the venous phase of the VA at the distal portion of its junction with the posterior inferior cerebellar artery (Figure 2C). These findings led to a diagnosis of dissecting aneurysm. Although the character of her headache was severe intensity, the pain did started gradually, not suddenly, and was not of sharp quality as the typical pain of dissection. That was why, although its clinical onset of this VA dissection had remained

unknown, we posit that the etiology of her headache was different from the dissection, which we decided to treat conservatively.

Total tumor removal was via the suboccipital retrosigmoid approach. Intraoperatively, the intramural hematoma of the dissecting aneurysm appeared to be old and unruptured, and we left it untreated. Cerebellar swelling was observed. The surgical specimen was a meningotheliomatous meningioma with internal hemorrhage. The next day, the patient fell into a stupor and computed tomography scans

(A)

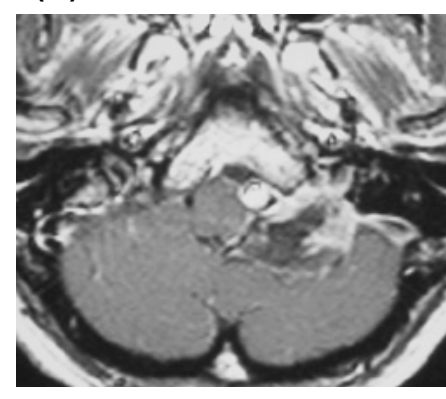

Figure 1: T1-weighted image with contrast medium on Magnetic resonance imaging showed a marked enhancement of tumor attached to the left jugular tubercle and hypo-intensity area, suggesting a meningioma with an intratumoral hemorrhage. Beside the tumor, left vertebral artery (VA) showed the hyper-intensity area along with the flow-void signal, suggesting intramural hemorrhage of dissecting aneurysm (A; axial image, B; coronal image).

*Corresponding author: Yasuhiko Hayashi, MD, Department of Neurosurgery Graduate School of Medical Science, Kanazawa University, 13-1, Takara-machi, Kanazawa, 920-8641, Japan, Tel: +81-76-265-2384; Fax: +81-76-234-4262; E-mail: yahayashi@ns.m.kanazawa-u.ac.jp

Received April 05, 2012; Accepted May 26, 2012; Published May 28, 2012

Citation: Hayashi Y, Kimura M, Kinoshita A, Hamada JI (2012) Occlusion of an Incidentally-Found Dissecting Aneurysm of the Vertebral Artery at Removal of a Jugular Tubercle Meningioma. Brain Disorders Ther 1:102. doi:10.4172/2168975X.1000102

Copyright: @ 2012 Hayashi $\mathrm{Y}$, et al. This is an open-access article distributed under the terms of the Creative Commons Attribution License, which permits unrestricted use, distribution, and reproduction in any medium, provided the original author and source are credited. 
Citation: Hayashi Y, Kimura M, Kinoshita A, Hamada JI (2012) Occlusion of an Incidentally-Found Dissecting Aneurysm of the Vertebral Artery at Removal of a Jugular Tubercle Meningioma. Brain Disorders Ther 1:102. doi:10.4172/2168-975X.1000102

(A)

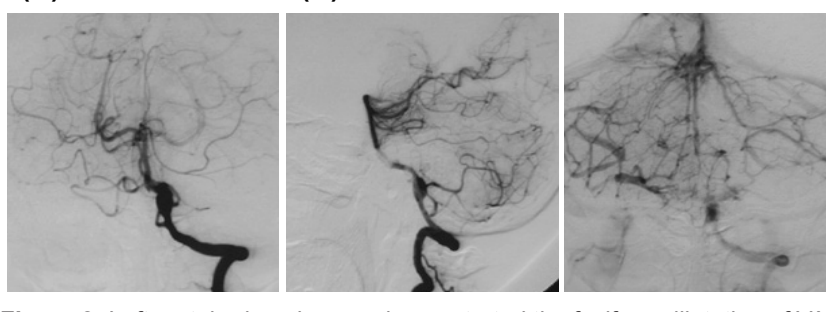

Figure 2: Left vertebral angiogram demonstrated the fusiform dilatation of VA in the arterial phase $(A$; anterior-posterior view, $B$; lateral view) and the retention of contrast medium in the venous phase $(C)$.

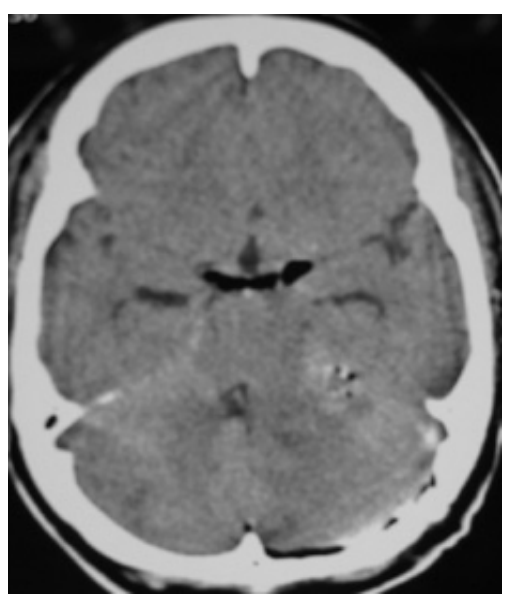

Figure 3: Computed tomography scan disclosed the disappearance of quadrigeminal cistern and compression of midbrain by vermis, indicating the upward herniation of cerebellar vermis.
(A)

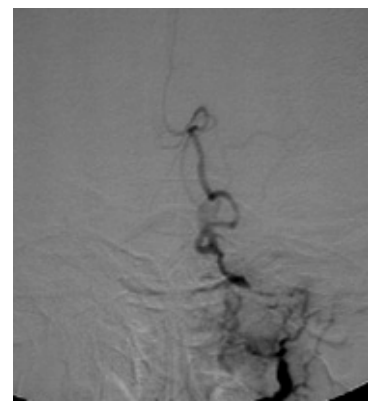

(B)

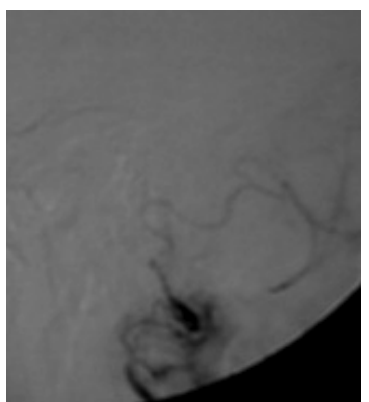

Figure 4: Left vertebral angiograpm demonstrated the occlusion of VA and its dissecting aneurysm (A; anterior-posterior view, B; lateral view).

revealed the disappearance of the left quadrigeminal cistern, indicative of upward herniation of the vermis (Figure 3). We immediately performed extensive decompressive suboccipital craniectomy and she regained consciousness. Follow-up angiography one month later disclosed occlusion of the left VA and the dissecting aneurysm (Figure 4). Her neurological status improved gradually and one month later she was transferred to a rehabilitation center.

\section{Discussion}

The diagnosis of dissecting aneurysm is usually based on angiographic findings. Hyper-intensity along a flow void sign on T1-weighted MRI suggests intramural hematoma and is helpful in reaching an accurate diagnosis [5-7].

We made our diagnosis because the angiogram showed a fusiform dilatation and contrast retention. Although we incidentally found a dissecting aneurysm on preoperative angiographs and MRI scans, we posit its asymptomatic pre-existence, because there were no previous episodes severe headache, and no evidence of subarachnoid haemorrhage (SAH) or infarction on MRI. As a small percentage of patients with dissection is reported asymptomatic in the absence of evidence of SAH or infarction, the recommended treatment is conservative therapy $[4,5]$.

Our patient manifested upward herniation after the operation. We posit that this may be attributable to cerebellar swelling associated with intratumoral hemorrhage and retraction of the peritumoral edematous brain. We also hypothesize that intraoperative manipulation resulted in the occlusion of the dissecting aneurysm and led to subsequent upward herniation.

Haemodynamic changes in the VA and dissecting aneurysm may lead to thrombogenesis and subsequent occlusion $[4,8]$. Therefore, if a dissecting, asymptomatic aneurysm is found incidentally, great care must be taken not to exacerbate brain edema.

\section{References}

1. Berger MS, Wilson CB (1984) Intracranial dissecting aneurysms of the posterio circulation. Report of six cases and review of the literature. J Neurosurg 61: 882-894.

2. Kitanaka C, Sasaki T, Eguchi T, Teraoka A, Nakane M, et al. (1994) Intracranial vertebral artery dissections: clinical, radiological features, and surgical considerations. Neurosurgery 34: 620-626.

3. Kitanaka C, Tanaka J, Kuwahara M, Teraoka A, Sasaki T, et al. (1994) Nonsurgical treatment of unruptured intracranial vertebral artery dissection with serial follow-up angiography. J Neurosurg 80: 667-674.

4. Schievink WI (2001) Spontaneous dissection of the carotid and vertebra arteries. N Engl J Med 344: 898-906.

5. Nakagawa K, Touho H, Morisako T, Osaka Y, Tatsuzawa K, et al. (2000) Longterm follow-up study of unruptured vertebral artery dissection: clinical outcomes and serial angiographic findings. J Neurosurg 93: 19-25.

6. Yoshimoto Y, Wakai S (1997) Unruptured intracranial vertebral artery dissection. Clinical course and serial radiographic imagings. Stroke 28: 370-374.

7. Arnold M, Bousser MG, Fahrni G, Fischer U, Georgiadis D, et al. (2006) Vertebral artery dissection: presenting findings and predictors of outcome. Stroke 37: 2499-2503.

8. Hayashi T, Hirose Y, Sagoh M, Murakami H (2000) Spontaneous occlusion of ruptured vertebral artery dissection at the extradural fenestration associated with extradural origin of the posterior inferior cerebellar artery--case report Neurol Med Chir (Tokyo) 40: 164-168. 